\title{
CUIDADO DE ENFERMAGEM A FAMILIARES DE PACIENTES EM UNIDADE DE TERAPIA INTENSIVA: REVISÃO INTEGRATIVA
}

\section{Tatiana Brusamarello}

Enfermeira. Doutora em Enfermagem. Coordenadora do Eixo Profissional de Enfermagem do Programa Residencia Integrada Multiprofissional em Atenção Hospitalar do Complexo Hospital de Clínicas da Universidade Federal do Paraná - UFPR, Brasil.

\section{Samantha Soares da Silva}

Enfermeira. Residente do Programa de Residência Multiprofissional em Urgência e Emergência do Complexo do Hospital de Clínicas - Universidade Federal do Paraná - UFPR, Brasil.

\section{Edineia Miranda Machado}

Enfermeira. Residente do Programa de Residência Multiprofissional em Urgência e Emergência do Complexo do Hospital de Clínicas -Universidade Federal do Paraná - UFPR, Brasil
Autor correspondente:

Maria Camila Buarraj Gomes

maria-camila@hmcp.puc-campinas.edu.br
RESUMO: Este estudo teve como objetivo identificar o conhecimento produzido sobre o cuidado de enfermagem a familiares de pacientes adultos em unidade de terapia intensiva. Trata-se de uma revisão integrativa, realizada em novembro de 2018 nas bases de dados MEDLINE, LILACS e SCOPUS. Os critérios de inclusão foram artigos em português, inglês e espanhol, disponíveis gratuitamente na íntegra e que respondessem à pergunta de pesquisa. Os dados dos 25 artigos selecionados foram analisados qualitativamente de acordo com a análise temática. Emergiram as seguintes categorias: estratégias exitosas no acolhimento de familiares na UTI; dificuldades encontradas para realizar o cuidado aos familiares na UTI; e valorização do cuidado humanizado aos familiares na UTI. Conclui-se que o cuidado dispensado as famílias nas unidades de terapia intensiva ainda é frágil. No entanto, as experiências exitosas de alguns enfermeiros promoveram ambiente de segurança e confiança entre a equipe de saúde e os familiares.

PALAVRAS-CHAVE: Cuidados de enfermagem; Família; Unidade de terapia intensiva.

\section{NURSING CARE TO RELATIVES OF PATIENTS IN INTENSIVE CARE UNITS: INTEGRATIVE REVIEW}

\begin{abstract}
Knowledge on nursing care with patients' relatives in an intensive care unit is identified through an integrative review undertaken in November 2018 and retrieved from databases MEDLINE, LILACS and SCOPUS. Inclusion criteria were articles in Portuguese, English and Spanish, freely available, in full, and on the research theme. Data of the 25 articles were analyzed qualitatively according to the theme. The following categories were selected: successful strategies in welcoming relatives in the ICU, difficulties in the care of relatives in the ICU, valorization of humanized care given to relatives in ICU. Results show that care to families in ICUs is still slight, even though there are successful experiences of several nurses who promoted a positive and confident environment between the health team and relatives.
\end{abstract}

KEY WORDS: Nursing Care; Family; Intensive Care Units.

\section{INTRODUÇÃO}

A família exerce papel de colaboradora no processo de tratamento e recuperação da saúde de seus entes. Desta forma, precisa ser considerada como uma parte integrante do cuidado realizado pela equipe de saúde ${ }^{1}$.

Assim, cabe aos profissionais de saúde a compressão de que a doença, seja em sua forma aguda ou crônica, apresenta ameaça tanto 
à integridade pessoal e familiar de quem adoece, fato agravado quando há a necessidade de uma hospitalização, principalmente, quando inesperada $a^{1-2}$. Neste sentido, alguns autores referem que, a doença, internação hospitalar e incerteza do restabelecimento do estado de saúde, produzam sofrimento, medo e alteração da vida cotidiana dos envolvidos ${ }^{1,3}$.

No ano de 2003, foi lançado pelo Ministério da Saúde a Política Nacional de Humanização do Sistema Único de Saúde, conhecida também como o HumanizaSUS, a fim de fortalecer os princípios do SUS no cotidiano das práticas de atenção e gestão, qualificando a saúde pública no Brasil e incentivar a comunicação e a troca de experiências entre os gestores, trabalhadores e usuários ${ }^{4}$.

Dentre as propostas do HumanizaSUS, a visita aberta passa a ser um direito do acompanhante, possibilitando aos familiares a permanência junto ao seu familiar, por um período mais longo, em episódio de internação hospitalar ${ }^{4}$. Nas Unidades de Terapia Intensiva adulto, geralmente a atenção dos familiares fica centrada na condição de ameaça à vida de seu parente e na evolução do seu estado de saúde. O que implica em ações que produzem desconforto para com elas mesmas, como não se alimentar, não conseguir descansar, menosprezar os próprios problemas de saúde e verbalizar pouco suas angústias e medos 5 .

Muitos encaram o ambiente crítico como um ambiente hostil, que gera medos e angústias, e frente a isso a equipe com conhecimentos e estratégias pode dirimir a tensão e favorecer a uma expectativa positiva de que esta fase seja encarada de uma forma mais leve e construtiva para ser ofertado o melhor tratamento possível para este paciente e seus familiares. Destaca-se que as equipes de saúde têm como princípio oferecer ao paciente o cuidado integral, atuando como promotores da integridade da vida, e promover a corresponsabilização dos familiares no processo do cuidado e reabilitação ${ }^{6}$.

Mediante o exposto este estudo tem como objetivo identificar o conhecimento produzido sobre o cuidado de enfermagem a familiares de pacientes adultos em unidade de terapia intensiva.

\section{METODOLOGIA}

Trata-se de uma revisão integrativa da literatura para qual foram seguidas seis fases: 1 . seleção das hipóteses ou questões da revisão; 2. demonstração da pesquisa a ser revista; 3. representação das características do estudo e suas constatações; 4 . análise das constatações; 5. interpretação dos resultados; e 6. relato da revisão. ${ }^{7}$

$\mathrm{Na}$ primeira fase, a fim de conduzir a busca para o alcance do objetivo proposto, foi formulada a seguinte questão norteadora: Como a enfermagem realiza o cuidado aos familiares de pacientes adultos internados em UTI?

Para responder esta questão foi realizada no mês de novembro de 2018, uma busca nas seguintes bases de dados: LILACS, MEDLINE e SCOPUS. Para tanto utilizouse a seguinte estratégia de busca, com descritores selecionados a partir dos descritores: "nursing care" AND ("profissional-family relations" OR "family") AND "intensive care units".

Constituíram-se critérios de inclusão artigos em português, inglês e espanhol, publicados de janeiro de 2008 a outubro de 2018, disponíveis na íntegra gratuitamente. Foram excluídos artigos de revisão, editoriais e texto que não respondessem à questão do estudo.

Dos 396 artigos encontrados, 249 foram da MEDLINE, 89 na LILACS e 58 na SCOPUS. Após exclusão de artigos repetidos procedeu-se à leitura dos títulos e resumos com objetivo de selecionar aqueles que pudessem vir a responder à questão do estudo. A partir desta seleção procedeu-se à leitura dos artigos na íntegra.

A amostra final resultou em 25 artigos. As etapas da seleção dos artigos foram executadas por dois pesquisadores, de forma independente e estão representadas na Figura 1.

Para coleta dos dados principais dos artigos foi construído pelas pesquisadoras um instrumento contendo os seguintes itens: título do artigo; nome do periódico, autores, país, idioma, ano de publicação, base de dados, cuidados de enfermagem realizados aos familiares adultos de pacientes internados em UTI. 


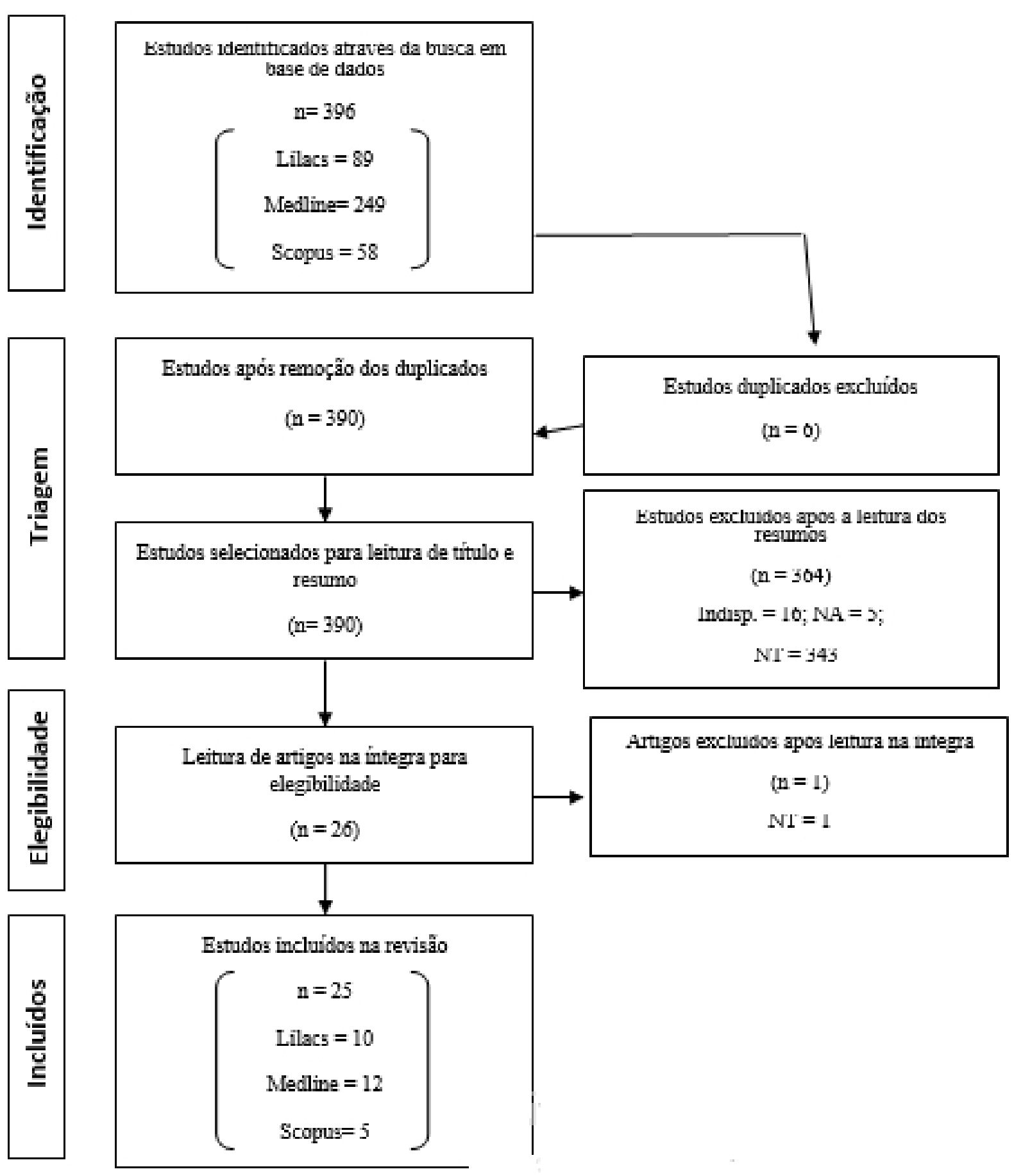

Figura 1. Fluxograma das etapas metodológicas da revisão. Curitiba, PR, Brasil, 2018.

Legenda: NA = não é artigo; NT = não é da temática; Indisp. = Artigo indisponível eletronicamente na íntegra.

A análise dos dados foi realizada de acordo com a proposta de análise temática ${ }^{8}$ composta por três etapas: pré-análise, exploração do material e tratamento dos resultados e, interpretação. 


\section{RESULTADOS}

Dos 25 artigos que compuseram este estudo, cinco foram publicados em 2010, cinco em 2009, quatro em 2014, três em 2012, dois em 2008 e 2016 e um em 2015 e 2018.

Quanto aos locais de publicação o Brasil se destacou com 15 estudos publicados, seguido pelos Estados Unidos da América com seis.

Após a leitura e análise dos resultados emergiram três categorias temáticas, sendo estas: estratégias exitosas no acolhimento de familiares na UTI; dificuldades encontradas para realizar o cuidado aos familiares na UTI; e valorização do cuidado humanizado aos familiares na UTI.

Quadro 1. Características dos estudos incluídos na categoria temática de cuidado de enfermagem aos familiares de pacientes internados em UTI, Curitiba, Paraná, 2019

\begin{tabular}{|c|c|c|c|c|c|}
\hline Id. & Título & Autores & Revista & País & Ano \\
\hline 1 & $\begin{array}{l}\text { Implementation of the evidence for the improvement } \\
\text { of nursing care to the critical patient's family: a } \\
\text { Participatory Action Research. }\end{array}$ & $\begin{array}{c}\text { de-la-Cueva-Ariza L, et } \\
\text { al. }{ }^{9}\end{array}$ & $\begin{array}{l}\text { BMC Health Services } \\
\text { Research }\end{array}$ & Espanha & 2018 \\
\hline 2 & $\begin{array}{l}\text { Percepções e necessidades dos familiares de pacientes } \\
\text { internados em uma unidade cardiológica intensiva }\end{array}$ & Camponogara S, et al..$^{10}$ & $\begin{array}{l}\text { Revista Mineira de } \\
\text { Enfermagem }\end{array}$ & Brasil & 2016 \\
\hline 3 & $\begin{array}{l}\text { O cotidiano de familiares de pacientes internados na } \\
\text { UTI: um estudo com as representações sociais }\end{array}$ & Vasconcelos EV, et al. ${ }^{11}$ & $\begin{array}{l}\text { Cuidado é } \\
\text { Fundamental }\end{array}$ & Brasil & 2016 \\
\hline 4 & $\begin{array}{l}\text { O acolhimento no cuidado à família numa unidade de } \\
\text { terapia intensiva }\end{array}$ & Passos SSS, et al. ${ }^{12}$ & $\begin{array}{l}\text { Revista Enfermagem } \\
\text { UERJ }\end{array}$ & Brasil & 2015 \\
\hline 5 & $\begin{array}{l}\text { Percepção da comunicação, satisfação e necessidades } \\
\text { dos familiares em Unidade de Terapia Intensiva }\end{array}$ & Puggina AC, et al. ${ }^{13}$ & $\begin{array}{l}\text { Escola Anna } \\
\text { Nery Revista de } \\
\text { Enfermagem }\end{array}$ & Brasil & 2014 \\
\hline 6 & $\begin{array}{c}\text { Factors affecting the nurse-patients' family } \\
\text { communication in intensive care unit of kerman: a } \\
\text { qualitative study. }\end{array}$ & $\begin{array}{l}\text { Loghmani L; Borhani F, } \\
\text { Abbaszadeh A. }{ }^{14}\end{array}$ & $\begin{array}{l}\text { Journal ofCaring } \\
\text { Sciences }\end{array}$ & Irã & 2014 \\
\hline 7 & $\begin{array}{l}\text { Family Visitation in Greek Intensive Care Units: Nurses' } \\
\text { Perspective }\end{array}$ & Athanasiou A, et al. ${ }^{15}$ & $\begin{array}{l}\text { American Journal of } \\
\text { Critical Care }\end{array}$ & Grécia & 2014 \\
\hline 8 & $\begin{array}{l}\text { Open intensive care units: a global challenge for } \\
\text { patients, relatives, and critical care teams }\end{array}$ & Cappellini E, et al. ${ }^{16}$ & $\begin{array}{l}\text { Dimensions of } \\
\text { Critical Care Nursing }\end{array}$ & EUA & 2014 \\
\hline 9 & $\begin{array}{l}\text { O impacto da visita de enfermagem sobre as } \\
\text { necessidades dos familiares de pacientes de UTI }\end{array}$ & Simoni RCM, et al. ${ }^{17}$ & $\begin{array}{l}\text { Revista da Escola de } \\
\text { Enfermagem da USP }\end{array}$ & Brasil & 2012 \\
\hline 10 & $\begin{array}{c}\text { Avaliação das estratégias de acolhimento na Unidade de } \\
\text { Terapia Intensiva. }\end{array}$ & Maestri E, et al. ${ }^{18}$ & $\begin{array}{l}\text { Revista da Escola de } \\
\text { Enfermagem da USP }\end{array}$ & Brasil & 2012 \\
\hline 11 & $\begin{array}{c}\text { Alterações na dinâmica familiar com a hospitalização } \\
\text { em unidade de terapia intensiva }\end{array}$ & Sell CT, et al. ${ }^{19}$ & $\begin{array}{l}\text { Revista Enfermagem } \\
\text { UERJ }\end{array}$ & Brasil & 2012 \\
\hline 12 & $\begin{array}{l}\text { Familiares na sala de espera de uma Unidade de Terapia } \\
\text { Intensiva: sentimentos revelados }\end{array}$ & Frizon $\mathrm{G}$, et al. ${ }^{20}$ & $\begin{array}{l}\text { Revista Gaúcha de } \\
\text { Enfermagem }\end{array}$ & Brasil & 2011 \\
\hline 13 & $\begin{array}{c}\text { Cuidando do paciente no processo de morte na } \\
\text { Unidade de Terapia Intensiva }\end{array}$ & $\begin{array}{l}\text { Da Silva RS; Campos } \\
\text { AER; Pereira A. }{ }^{21}\end{array}$ & $\begin{array}{l}\text { Revista da Escola de } \\
\text { Enfermagem da USP }\end{array}$ & Brasil & 2011 \\
\hline 14 & $\begin{array}{c}\text { Grupo de suporte como estratégia para acolhimento } \\
\text { de familiares de pacientes em Unidade de Terapia } \\
\text { Intensiva }\end{array}$ & Oliveira LMAC, et al. ${ }^{22}$ & $\begin{array}{l}\text { Revista da Escola de } \\
\text { Enfermagem da USP }\end{array}$ & Brasil & 2010 \\
\hline
\end{tabular}


(Conclusão)

\begin{tabular}{|c|c|c|c|c|c|}
\hline Id. & Título & Autores & Revista & País & Ano \\
\hline 15 & $\begin{array}{l}\text { The effect of a family support intervention on family } \\
\text { satisfaction, length-of-stay, and cost of care in the } \\
\text { intensive care unit }\end{array}$ & Shelton $\mathbb{W}$, et al. ${ }^{23}$ & $\begin{array}{l}\text { Critical Care } \\
\text { Medicine }\end{array}$ & EUA & 2010 \\
\hline 16 & $\begin{array}{l}\text { Effectiveness trial of an intensive communication } \\
\text { structure for families of long-stay ICU patients }\end{array}$ & Daly BJ, et al. ${ }^{24}$ & $\begin{array}{l}\text { American College of } \\
\text { Chest Physicians }\end{array}$ & EUA & 2010 \\
\hline 17 & $\begin{array}{l}\text { Expectativas de familiares de clientes em UTI sobre o } \\
\text { atendimento em saúde: estudo sociopoético }\end{array}$ & $\begin{array}{l}\text { Da Silva FS; Dos Santos } \\
\text { I. }{ }^{25}\end{array}$ & $\begin{array}{l}\text { Escola Anna } \\
\text { Nery Revista de } \\
\text { Enfermagem }\end{array}$ & Brasil & 2010 \\
\hline 18 & $\begin{array}{l}\text { Embrace hope: an end-of-life intervention to support } \\
\text { neurological critical care patients and their families. }\end{array}$ & Yeager $S$, et al ${ }^{26}$ & Critical Care Nurse & EUA & 2010 \\
\hline 19 & $\begin{array}{l}\text { Comunicação da equipe de enfermagem com a família } \\
\text { do paciente. }\end{array}$ & $\begin{array}{l}\text { Casanova EG; Lopes } \\
\text { GT. }{ }^{27}\end{array}$ & $\begin{array}{l}\text { Revista Brasileira de } \\
\text { Enfermagem }\end{array}$ & Brasil & 2009 \\
\hline 20 & $\begin{array}{c}\text { Death, empathy and self preservation: the emotional } \\
\text { labour of caring for families of the critically ill in adult } \\
\text { intensive care }\end{array}$ & Stayt LC. ${ }^{28}$ & $\begin{array}{l}\text { Journal of Clinical } \\
\text { Nursing }\end{array}$ & $\begin{array}{l}\text { Reino } \\
\text { Unido }\end{array}$ & 2009 \\
\hline 21 & $\begin{array}{l}\text { Symptoms experienced by family members of patients } \\
\text { in intensive care units. }\end{array}$ & Mcadam JL; Puntillo K. ${ }^{29}$ & $\begin{array}{l}\text { American Journal of } \\
\text { Critical Care }\end{array}$ & EUA & 2009 \\
\hline 22 & $\begin{array}{l}\text { Registered nurses as family care specialists in the } \\
\text { intensive care unit. }\end{array}$ & Nelson DP; Plost G. ${ }^{30}$ & Critical Care Nurse & EUA & 2009 \\
\hline 23 & $\begin{array}{l}\text { Internação em Unidade de Terapia Intensiva e a família: } \\
\text { perspectivas de cuidado }\end{array}$ & $\begin{array}{l}\text { Bettinelli LA; Erdmann } \\
\qquad \text { AL. }^{31}\end{array}$ & $\begin{array}{l}\text { Avances en } \\
\text { Enfermería }\end{array}$ & Brasil & 2009 \\
\hline 24 & $\begin{array}{l}\text { Os significados nas mensagens dos familiares de } \\
\text { pacientes em estado de coma internados em UTI. }\end{array}$ & $\begin{array}{l}\text { Puggina ACG; Da Silva } \\
\text { MJP; Araújo MMT. }{ }^{32}\end{array}$ & $\begin{array}{l}\text { Acta Paulista de } \\
\text { Enfermagem }\end{array}$ & Brasil & 2008 \\
\hline 25 & $\begin{array}{l}\text { Ansiedade e depressão em familiares de pacientes } \\
\text { internados em unidade de cuidados intensivos }\end{array}$ & $\begin{array}{l}\text { Maruiti MR; Galdeano } \\
\text { LE; Farah OGD. }\end{array}$ & $\begin{array}{l}\text { Acta Paulista de } \\
\text { Enfermagem }\end{array}$ & Brasil & 2008 \\
\hline
\end{tabular}

Fonte: Dados da pesquisa, 2019.

\section{ESTRATÉGIAS EXITOSAS NO ACOLHIMENTO DE FAMILIARES NA UTI}

Os artigos analisados apresentaram diferentes estratégias para realizar o acolhimento dos familiares de pacientes internados na UTI, destacam-se as visitas de enfermagem, criação de grupos de suporte, flexibilidade de horário e de número de visitantes, bem como a permissão para apoio espiritual.

As Visitas de Enfermagem foram realizadas em três momentos consecutivos da internação do paciente na UTI o que possibilitou mensurar e trabalhar a carga emocional e as principais dúvidas da família durante esse período, também contribui para detectar e prevenir precocemente sintomas de ansiedade, depressão e estresse vivenciados pelos familiares ${ }^{17: 69}$.
A possibilidade de flexibilidade quanto ao horário e ao número de visitantes da UTI também é uma boa estratégia de acolhimento, porque os familiares se sentem amparados, ajudados, compreendidos e confortados $^{18: 79}$.

O uso do grupo de suporte [...] destinado ao atendimento de familiares de pacientes internados nas UTIs [...] é um recurso terapêutico que pode colaborar para a construção de uma prática assistencial humanizada e acolhedora que promova 0 respeito às pessoas, possibilitando a superação do olhar na doença $a^{22: 430 ; 436}$.

O cuidado espiritual e o suporte emocional foi considerado como um dos principais fatores contribuintes para atender às necessidades familiares dos pacientes, além de fortalecer as interações entre familiares e enfermeiros ${ }^{14: 77}$. 
DIFICULDADES ENCONTRADAS PARA REALIZAR O CUIDADO AOS FAMILIARES NA UTI

Nos textos publicados, foi possível observar que a escuta e a fala dispensadas aos familiares de paciente internados no ambiente crítico, são ações muitas vezes negligenciadas pelas equipes de saúde que atuam nos ambientes críticos. A sobrecarga de atividades e as excessivas horas de jornada de trabalho também foram apontadas como complicadores na hora de desenvolver ações para atender as famílias na UTI. Ainda, a gravidade do quadro clínico do paciente exige dos profissionais maior atenção na realização da assistência, em contrapartida o estado emocional dos seus familiares acaba sendo menosprezado.

[...] a abertura à família é frágil, talvez em virtude, dentre outros fatores, do pouco preparo dos trabalhadores que atuam nas UTIs para o atendimento a essa clientela de familiares, aliado à rotina complexa destas unidades, que muitas vezes faz com que os profissionais da saúde ignorem ou deixem de utilizar em suas ações atos como o tocar, conversar, ouvir, em detrimento da demanda de atividades que necessitam realiza"20:77.

Com a sobrecarga de trabalho e as excessivas horas de jornada, as enfermeiras afastamse dos familiares, tentando falar apenas o necessário $^{12: 371}$.

Por se tratar de um ambiente no qual a gravidade dos pacientes se faz presente, percebe-se que a maioria dos trabalhadores de enfermagem acaba por valorizar, em excesso, o estado físico do paciente em detrimento do estado emocional do mesmo e de seus familiares ${ }^{10: 6}$.

\section{VALORIZAÇÃO DO CUIDADO HUMANIZADO AOS} FAMILIARES NA UTI

Nos textos analisados, ficaram evidente os resultados positivos do melhor acolhimento aos familiares na UTI, possibilitando a estes melhor compreensão do estado de saúde dos pacientes críticos internados. Ainda, destaca-se o fato de que a compreensão do estado de saúde do seu familiar contribui para minimização do sofrimento frente ao processo de morte e morrer.
Também, foi possível observar a necessidade dos profissionais de saúde se aperfeiçoarem nesta temática e assim poderem construir estratégias para cuidarem de forma mais abrangente dos familiares.

É fundamental não só, mas principalmente dentro de uma UTI, compreender a família como extensão do doente. [...] os familiares foram citados como necessitados dos cuidados de enfermagem para melhor compreenderem e minimizar o seu sofrimento diante do processo de morte $\mathrm{e}$ $\operatorname{morrer}^{21: 742}$.

É necessário é indispensável o aperfeiçoamento dos profissionais para dar maior apoio e demonstrar solidariedade nos momentos de sofrimento ${ }^{31: 18}$.

\section{DISCUSSÃO}

A humanização e o acolhimento são temas de discussão amplos e relevantes para os profissionais de saúde, entre estes destaca-se a equipe de enfermagem, que presta cuidado direto e contínuo ao paciente. Assim, ao considerar a fragilidade e complexidade que os pacientes e familiares vivenciam com o processo de internação na UTI é possível mensurar a importância de valorizar e promover discussão sobre possibilidades de ações que visem implantar diferentes formas de atender esta população.

Destaca-se que um dos objetivos do HumanizaSUS é incentivar a participação ativa de pacientes e familiares no contexto do cuidado realizado nos diferentes serviços de saúde ${ }^{4}$. Neste sentido, ressalta-se que as estratégias encontradas nesta revisão estão em concordância com a PNH, pois envolvem técnicas de acolhimento e comunicação com os familiares.

A comunicação foi apontada como ferramenta essencial para o desenvolvimento de qualquer ação envolvida no cuidado aos familiares de pacientes internados em UTI, pois, possibilita que o profissional de enfermagem proporcione apoio emocional, repasse de informações sobre normas e rotinas do serviço, explicação do quadro clínico e dos cuidados realizados ao paciente, entre outros ${ }^{14,20}$. No entanto, os textos analisados, referenciaram que algumas equipes de enfermagem atuantes em ambientes críticos, muitas vezes 
se sentem sobrecarregadas pela gravidade do quadro clínico dos pacientes, de modo a priorizar os cuidados técnicos, praticando uma comunicação mecânica e pouco humanizada, com utilização excessiva de termos técnicos nas conversas com os familiares, potencializando a barreira na comunicação entre os envolvidos ${ }^{10,27}$.

A visita de enfermagem ao familiar foi apontada como uma oportunidade do enfermeiro se colocar à disposição, destacando-se como uma abordagem efetiva, por fornecer aos familiares acolhimento e informações de forma direcionada e compreensível. Esta estratégia possibilita que o enfermeiro detecte as principais necessidades do familiar, permitindo que trabalhe de forma preventiva, evitando o surgimento de sintomas negativos como, por exemplo: ansiedade, depressão e estresse $\mathrm{e}^{17,25}$.

A prática hospitalar, em especial a praticada em UTIs é normativa, controladora e hierarquizada ${ }^{31}$. Para o familiar o horário de visitas é um momento muito esperado, sendo uma oportunidade única no dia para rever o paciente. Entretanto, esse momento pode ser interpretado por familiares como um limitador que impossibilita o acesso irrestrito impossibilitando que transmita seu carinho e segurança ao paciente ${ }^{10}$.

A flexibilização no horário de visita e no número de pessoas é visto como uma estratégia efetiva de acolhimento, tanto para o paciente quanto para os familiares, que se sentem confortados por estarem em contato com o ente querido ${ }^{18}$. Emalguns casos, essafaltade flexibilidade pode impedir que o familiar visite o paciente, visto que o familiar pode não ter a disponibilidade de realizar a visita no momento estipulado pela instituição ${ }^{10}$. Neste sentido, um dos estudos destacou que em alguns casos os familiares apresentaram dificuldades para o deslocamento até o hospital por este ser oneroso e nem sempre viável ${ }^{27}$.

Alguns estudos trazem a experiência de UTI aberta aos familiares, porém a falta de evidências de benefícios reais aos pacientes, a instabilidade do paciente, as dificuldades vivenciadas pela equipe de enfermagem como, por exemplo, a sobrecarga de trabalho e falta de treinamento, são fatores que dificultam, e em algumas realidades inviabilizam a implementação dessa estratégia ${ }^{15-16}$.
O único método de abordagem coletiva encontrado nesta revisão foi o Grupo de Apoio aos Familiares (GRAF), que funciona como um espaço aberto em que o coordenador do grupo oferece informações gerais sobre o processo de internação na UTI desmistificando as ideias equivocadas dos familiares, permitindo a troca de experiências e esclarecendo os principais pontos de tensão. Trata-se de um recurso terapêutico que permite a prática assistencial humanizada, que promove a aproximação do enfermeiro com os familiares, a fim de conhecer e/ou suprir suas necessidades ${ }^{22}$.

Ainda, em relação a abordagens multidisciplinares foi encontrado o grupo Embrace Hope, este grupo atua acompanhando as famílias de pacientes em cuidados de fim de vida, atuam de forma estruturada proporcionando inúmeras intervenções para o acolhimento dos familiares, como exemplos: ferramentas de comunicação e salas preparadas para dar atualizações aos familiares em luto ${ }^{26}$.

Destaca-se que o enfermeiro é o profissional da equipe da UTI mais presente no cuidado, estando 24 horas em vigilância ininterrupta ao paciente, consequentemente é o profissional que está em maior contato com os familiares ${ }^{21}$. Apesar de o cuidado ao familiar ser uma atribuição de toda a equipe o enfermeiro é decisivo neste processo por ser o responsável pelo gerenciamento da unidade ${ }^{16}$, sendo assim, primordial que desenvolva capacidade para exercer o cuidado aos familiares ${ }^{10-11}$.

Em contrapartida, a complexidade dos cuidados que presta e a sobrecarga de trabalho combinadoà carência de profissionais, faz com que o enfermeiro priorize o que julga ser essencial ${ }^{12}$. Na UTI, manter o paciente estável é prioridade, sendo assim o enfermeiro tem a tendência a valorizar os cuidados técnicos incluindo a monitorização e o funcionamento adequado dos equipamentos de suporte que garantem a estabilidade do paciente, não percebendo a necessidade dos familiares aos cuidados de enfermagem ${ }^{10,20,27}$.

Por outro lado, ressalta-se que para o familiar o ambiente da UTI pode ser intimidador, por ser um local desconhecido onde observa o parente conectado a diversos equipamentos e passando por procedimentos desconhecidos, invasivos e dolorosos muitas vezes ${ }^{10}$. 
Assim, esta realidade apresenta-se como um processo complexo e de difícil enfrentamento para todos os envolvidos, pois desencadeia experiências negativas motivadas pela incerteza do futuro, principalmente pelo medo ou expectativa da perda ${ }^{11,18,20,27,29}$.

Todavia, o horário de visita na maioria das vezes é o único momento em que o familiar pode ter contato com o paciente internado, porém a falta de flexibilidade em relação ao tempo e orientações claras tornam este momento tenso. Entretanto, a maior parte das equipes de enfermagem das UTIs não está preparada para receber e cuidar dos familiares de forma efetiva $a^{10-11,16}$.

A falta de habilidade na comunicação do enfermeiro e da equipe de enfermagem somada a fragilidade das famílias e a complexidade do cuidado na UTI geram inúmeros conflitos entre profissionais e familiares. ${ }^{20}$ Fato corroborado pelo estudo que destacou que a discordância da família na escolha do tratamento e a não observância do horário de visita pelos familiares foram as principais causas de conflito ${ }^{14}$.

Os conflitos aumentam a tensão na relação entre o enfermeiro e os familiares traduzindo-se em resistência da equipe de enfermagem na interação com o familiar, sendo assim os enfermeiros criam estratégias para evitar o contato como, por exemplo: realizar os intervalos para alimentação no horário de visitas e ficar em silêncio durante a realização dos cuidados ${ }^{12}$.

A presença do familiar na UTI de fato aumenta a carga de trabalho do enfermeiro, o ambiente da UTI nem sempre é adequado para recepção de pessoas não envolvidas na assistência. No entanto, fica evidente que a falta de conhecimento do familiar sobre o quadro clínico do paciente, bem como a dificuldade no envolvimento com o tratamento de seu ente, pode gerar prejuízos aos pacientes $^{10}$. Deste modo fica clara a necessidade de preparo do enfermeiro para desenvolver diferentes estratégia para acolher e envolver os familiares neste ambiente complexo ${ }^{31,34}$.

\section{CONCLUSÃO}

Com este estudo foi possível expor a fragilidade vivenciada pelo familiar do paciente crítico e perceber a importância da sua inclusão no cuidado, porém apesar de existir na literatura diversas estratégias comprovadamente efetivas para execução desta ação, foi observado que em grande parte das UTIs este cuidado é negligenciado, sendo deixado em segundo plano ou até ignorado.

A comunicação foi observada como a ferramenta principal presente em todas as estratégias para o cuidado com o familiar, a partir das estratégias expostas nesta revisão é possível perceber que há diferentes maneiras de colocar este cuidado em prática, alinhando-as à realidade institucional e ao perfil dos usuários.

O cuidado com o familiar é centralizado na equipe de enfermagem, por serem os profissionais mais presentes no cuidado direto ao paciente, porém esta ação é de responsabilidade de toda equipe multidisciplinar. Por sua vez, as instituições também precisam oportunizar aos profissionais recursos para que a execução deste cuidado seja viável.

Entender o paciente e sua família como o elemento principal do cuidado é primordial para execução de um cuidado efetivo, integral e humanizado. Necessitase de mais estudos neste campo de conhecimento, visto que ainda é um problema comum em inúmeras UTIs.

\section{REFERÊNCIAS}

1. Freitas KS, Menezes IG, Mussi FC. Validação da escala de conforto para familiares de pessoas em estado crítico de saúde. Rev Latinoam Enferm. 2015; 23(4):660-8.

2. Bousso RS, Angelo M. Buscando preservar a integridade da unidade familiar: a família vivendo a experiência de ter um filho na UTI. Rev Esc Enferm USP. 2001; 35(2):172-9.

3. Oliveira R, Maruyama SAT. Princípio da integralidade numa UTI pública: espaço e relações entre profissionais de saúde e usuários. Rev Eletrônica Enferm. 2009; 11(2).

4. Ministério da Saúde. HumanizaSUS: Política nacional de humanização -humanização como eixo norteador das práticas de atuação e gestão em todas as esferas do SUS. 2003.

5. Horn EV, Tesh A. The effect of critical care hospitalization on family members: stress and responses.Dimens Crit Care Nur. 2000; 19(4):40-9. 
6. Ministério da Saúde, Secretariade Atenção à Saúde, Núcleo Técnicoda Política Nacionalde Humanização. HumanizaSUS: visita aberta e direito a acompanhante. 2007.

7. Ganong LH. Integrative reviews of nursing research. Research in nursing \& health. 1987; 10(1): $1-11$.

8. Bardin L. Análise de conteúdo. Lisboa: Editora Edições 70; 1977.

9. De-La-Cueva-Ariza L, et al. Implementation of the evidence for the improvement of nursing care to the critical patient's family: a Participatory Action Research. BMC Health Serv Res. 2018; 18(1): 357.

10. Camponogara S, et al. Percepções e necessidades dos familiares de pacientes internados em uma unidade cardiológica intensiva. REME rev min enferm. 2016; 20.

11. Vasconcelos EV, et al. O cotidiano de familiares de pacientes internados na UTI: um estudo com as representações sociais.Rev Pesqui Cuid Fundam. 2016; 8(2): 4313-27.

12. Passos SSS, et al. O acolhimento no cuidado à família numa unidade de terapia intensiva. Rev Enferm UERJ. 2015; 23(3): 368-74.

13. Puggina AC, et al. Percepção da comunicação, satisfação e necessidades dos familiares em Unidade de Terapia Intensiva. Esc Anna Nery Rev Enferm. 2014;18(2): 277-83.

14. Loghmani L, Borhani F, Abbaszadeh A. Factors affecting the nurse-patients' family communication in intensive care unit of kerman: a qualitative study. Journal of caring sciences. 2014; 3(1): 67.

15. Athanasiou A, et al. Family visitation in Greek intensive care units: nurses' perspective. Am J Crit Care. 2014; 23(4): 326-333.

16. Cappellini E, et al. Open intensive care units: a global challenge for patients, relatives, and critical care teams.Dimens Crit Care Nurs. 2014; 33(4): 18193.

17. Simoni RCM, et al. O impacto da visita de enfermagem sobre as necessidades dos familiares de pacientes de UTI. Rev Esc Enferm USP. 2012; 46: 65-70.

18. Maestri E, et al. Avaliação das estratégias de acolhimento na Unidade de Terapia Intensiva.Rev Esc Enferm USP. 2012; 46(1): 75-81.

19. Sell CT, et al. Alterações na dinâmica familiar com a hospitalização em unidade de terapia intensiva. Rev EnfermUERJ. 2012: 488-92.

20. Frizon $\mathrm{G}$, et al. Familiares na sala de espera de uma unidade de terapia intensiva: sentimentos revelados. Rev Gaúcha Enferm. 2011; 32(1): 72.

21. Silva RS, Campos AER, Pereira A. Cuidando do paciente no processo de morte na Unidade de Terapia Intensiva. 2011.

22. Oliveira LMAC, et al. Grupo de suporte como estratégia para acolhimento de familiares de pacientes em Unidade de Terapia Intensiva. Rev Esc Enferm USP. 2010; 44(2): 429-36.

23. Shelton $\mathrm{W}$, et al. The effect of a family support intervention on family satisfaction, length-of-stay, and cost of care in the intensive care unit. Crit Care Med. 2010; 38(5): 1315-20.

24. Daly BJ, et al. Effectiveness trial of an intensive communication structure for families of long-stay ICU patients. Chest. 2010; 138(6): 1340-48.

25. Silva FS, Santos I. Expectativas de familiares de clientes em UTI sobre o atendimento em saúde: estudo sociopoético. Esc Anna Nery Rev Enferm. 2010; 14(2): 5-230.

26. Yeager S, et al. Embrace hope: an end-of-life intervention to support neurological critical care patients and their families. Crit Care Nurse. 2010; 30(1): $47-58$.

27. Casanova EG, Lopes GT. Comunicação da equipe de enfermagem com a família do paciente. Rev Bras Enferm. 2009; 62(6).

28. Stayt LC. Death, empathy and self preservation: the emotional labour of caring for families of the critically ill in adult intensive care. J Clin Nur. 2009; 18(9): 1267-75.

29. Mcadam JL, Puntillo K. Symptoms experienced by family members of patients in intensive care units. Am J Crit Care. 2009; 18(3): 200-9.

30. Nelson DP, Plost G. Registered nurses as family care specialists in the intensive care unit. Crit Care Nurse. 2009; 29(3): 46-52.

31. Bettinelli LA, Erdmann AL. Internação em unidade 
de terapia intensiva e a família: perspectivas de cuidado. Av Enferm. 2009; 27(1): 15-21.

32. Puggina ACG, Silva MJP, Araújo MMT. Mensagens dos familiares de pacientes em estado de coma: a esperança como elemento comum. Acta Paul Enferm. 2008; 21(2).

33. Maruiti MR, Galdeano LG, Farah OGD. Ansiedade e depressão em familiares de pacientes internados em unidade de cuidados intensivos. Acta Paul Enferm. $2008 ; 21(4)$.

34. Zanetti TG, Graube SL, Dezordi CCM, Bittencourt VLL, Horn RC, Stumm EMF. Sintomas de estresse em familiares de pacientes adultos em terapia intensiva. Rev Saúde e Pesquisa. 2017; 10(3): 549-55.

Recebido em: 15/02/2019

Aceito em: 20/08/2019 